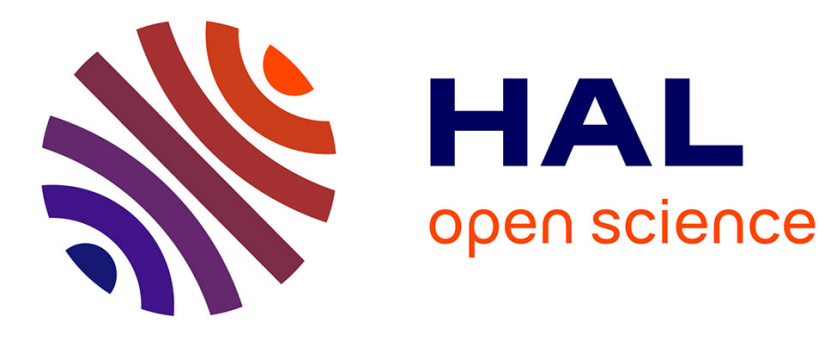

\title{
Un détecteur synchrone travaillant avec un relais
}

\author{
V. Maxia, G. Venturello
}

\section{To cite this version:}

V. Maxia, G. Venturello. Un détecteur synchrone travaillant avec un relais. Revue de Physique Appliquée, 1970, 5 (5), pp.777-778. 10.1051/rphysap:0197000505077700 . jpa-00243454

\section{HAL Id: jpa-00243454 https://hal.science/jpa-00243454}

Submitted on 1 Jan 1970

HAL is a multi-disciplinary open access archive for the deposit and dissemination of scientific research documents, whether they are published or not. The documents may come from teaching and research institutions in France or abroad, or from public or private research centers.
L'archive ouverte pluridisciplinaire HAL, est destinée au dépôt et à la diffusion de documents scientifiques de niveau recherche, publiés ou non, émanant des établissements d'enseignement et de recherche français ou étrangers, des laboratoires publics ou privés. 


\title{
UN DÉTECTEUR SYNCHRONE TRAVAILLANT AVEC UN RELAIS
}

\author{
V. MAXIA \\ Istituto di Fisica dell'Università, Cagliari, Italie \\ G. VENTURELLO \\ Istituto di Fisica dell'Università, Torino, Italie
}

(Reçu le 26 mai 1970)

\begin{abstract}
Résumé. - Dans cette note on décrit un détecteur à sensibilité de phase fonctionnant par un relais à mercure, dont les applications sont complémentaires à celles des circuits conventionnels. Il est en mesure de travailler correctement de basses fréquences jusqu'à $0,1 \mathrm{~Hz}$. Cependant il n'est pas indiqué pour fonctionner avec précision au-dessus de quelque dizaine de $\mathrm{Hz}$.
\end{abstract}

Abstract. - A phase-sensitive detector using a mercury relay is described, whose application are complementary to those of conventional circuits. It can work precisely at frequencies as low as $0.1 \mathrm{~Hz}$, even though being unable to behave correctly beyond some tens of $\mathrm{Hz}$.

On sait que les détecteurs synchrones communs travaillent correctement dans un intervalle de fréquence qui, en partant de quelques $\mathrm{Hz}$, ne descend jamais au-dessus de $1 \mathrm{~Hz}$ environ $[1,2,3]$. Néanmoins, dans certaines applications, il faudrait, à cause de la nature particulière du problème en question, employer des fréquences encore plus basses, par exemple $0,1 \mathrm{~Hz}$ ou encore moins. Clairement, la difficulté la plus considérable pour atteindre des fréquences aussi basses, dérive de la stabilité limitée du circuit électronique du détecteur [4]. Dans le but d'éviter cet obstacle nous avons projeté un détecteur synchrone, en utilisant un relais. En réalité, dans les travaux des pionniers sur le rejet du bruit on avait déjà usé des interrupteurs mécaniques $[5,6]$. Cependant ces dispositifs par la suite n'ont pas été développés de façon à jouir des excellentes prestations des relais modernes.

Dans la figure 1 on montre le circuit que nous avons utilisé. Le signal sinusoïdal de référence, traversant un circuit de déphasage approprié et un amplificateur saturé, alimente le bobinage du relais. Le montage est du type double $\left({ }^{1}\right)$ et fonctionne par des contacts à mercure qui permettent une fréquence maximale de $300 \mathrm{~Hz}$. Le signal d'entrée est appliqué directement au circuit intégrateur $\mathrm{RC}$ et on le renverse périodiquement selon la phase de la référence. L'amplificateur du canal de référence actionne le relais lorsque le signal de référence atteint $10 \mathrm{mV}$. Si la valeur de crête du signal de référence est de $1 \mathrm{~V}$, ceci correspond à un déphasage de moins de $1^{\circ}$. Le déphaseur RC a été projeté surtout pour corriger le retard sur la commande du relais. Pour cette raison il agit

(1) SEEN BNHWY 2 RT. seulement pour avancer la phase de la référence. Pour des fréquences de quelques dizaines $\mathrm{d}^{\prime} \mathrm{Hz}$ on peut utiliser les valeurs $C_{1}=1 \mu \mathrm{F}$ et $R_{1}=0,5 \mathrm{M} \Omega$ Lorsqu'il faut atteindre des fréquences de près de $0,1 \mathrm{~Hz}$, on demande des valeurs de $C_{1}$ plus élevées, jusqu'à $100 \mu \mathrm{F}$. Mais, au niveau de ces fréquences et au-dessous, on peut éliminer $C_{1}$ parce qu'il devient inutile de compenser le retard du relais, qui est bien petit en comparaison de la période du signal de référence $(\geqslant 10 \mathrm{~s})$. Comme premier stade de l'amplicateur d. c. on a utilisé un transistor a effect de champ dans le but d'obtenir l'impédance d'entrée élevée demandée pour le bon fonctionnement du déphaseur. La résistance du drainage doit être choisie de façon que le voltage sur l'émetteur du transistor BSX 12 soit légèrement plus petit que la tension de rupture de la diode Zener. De cette façon on atteint à la sensibilité de commande maximale. Il faut que la constante de temps de l'intégrateur soit au moins une dizaine de fois plus grande que la période du signal dans le but d'éviter les fluctuations du signal de sortie. Pour cette raison, on demande des valeurs de

$$
R \geqslant 10 \mathrm{M} \Omega \text { et } C \geqslant 10 \mu \mathrm{F}
$$

pour travailler à $0,1 \mathrm{~Hz}$.

Dans la figure 2 on montre le bon fonctionnement du détecteur à la fréquence de $30 \mathrm{~Hz}$. Le point de potentiel zéro qui apparaît dans la photo exécutée avec le signal de référence hors de phase, correspond au temps de commutation du relais. A des fréquences plus élevées $(\sim 50 \div 200 \mathrm{~Hz}$ ), au contraire, le comportement est moins satisfaisant, et ce à cause d'une certaine indécision dans l'obtention du contact.

Dans nos expériences, le voltage sur la capacité d'intégration a été mesuré par un voltmètre élec- 

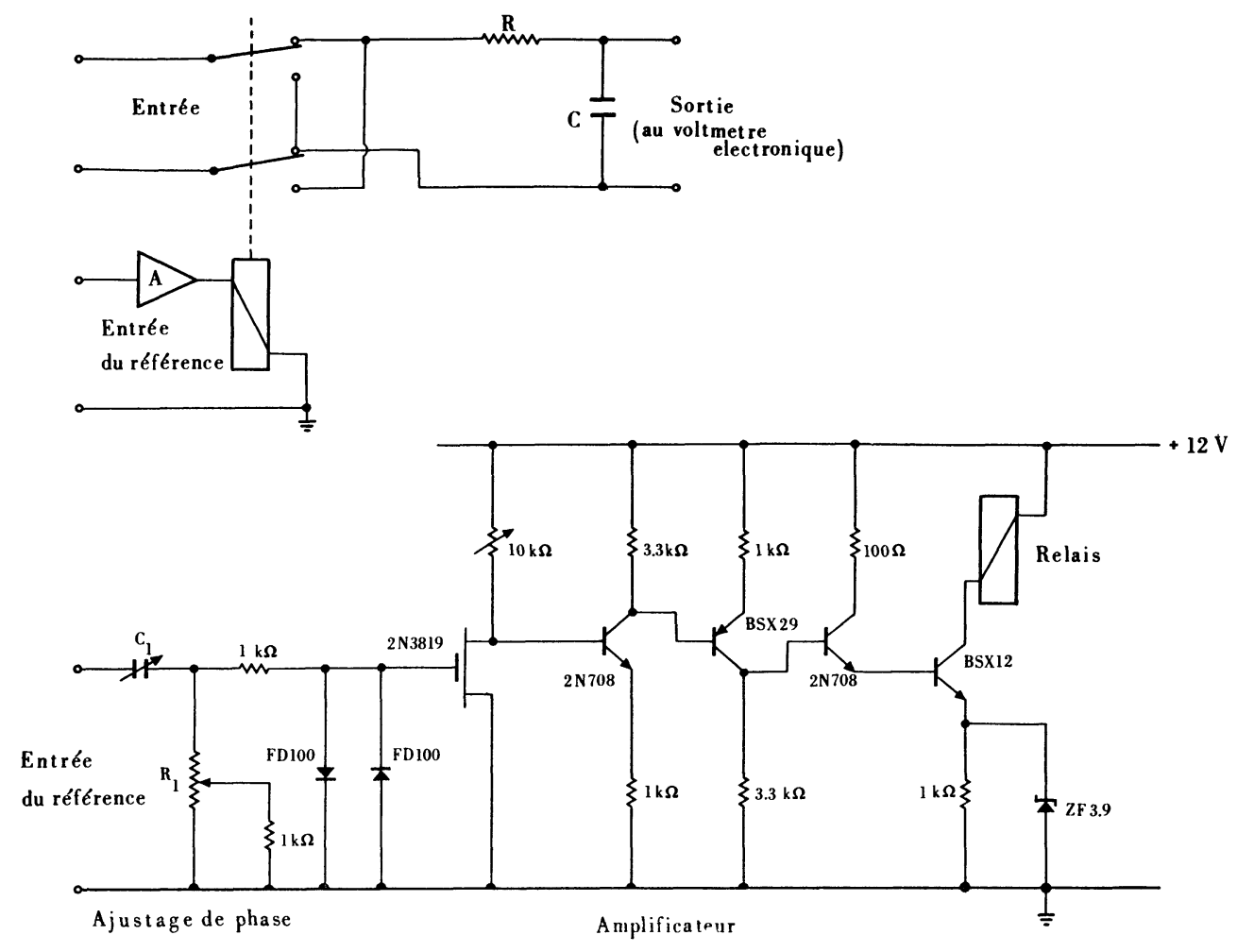

FIg. 1. - Circuit du détecteur synchrone.

tronique à forte impédance d'entrée. On a choisi un instrument à entrée flottante pour éviter des limitations dans les connexions du signal d'entrée. Dans ces

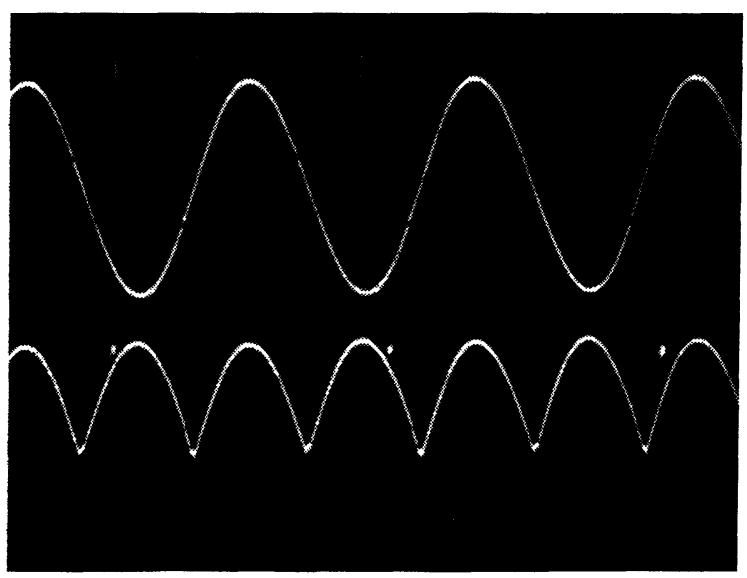

a) Référence et signal en phase. conditions on a constaté que l'unique difficulté réelle pour travailler à basse fréquence, provient des limites pratiques dans la durée du temps d'intégration.

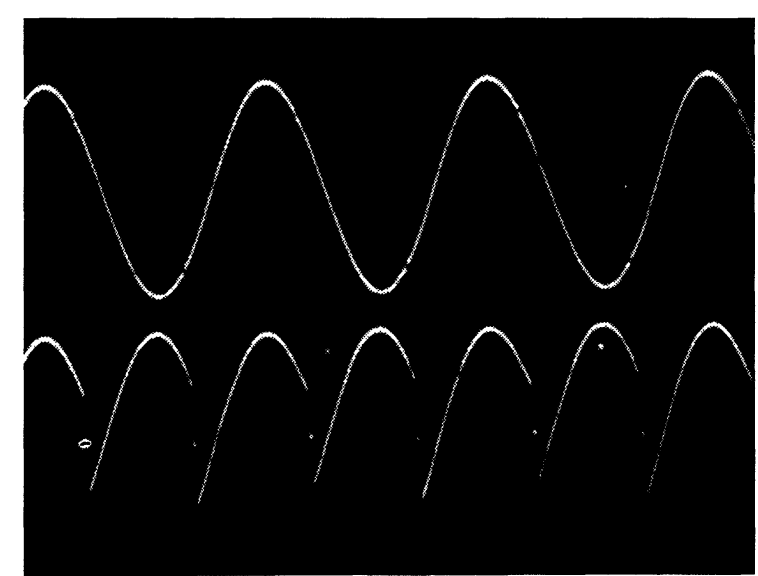

b) Référence déphasée en avance de $20^{\circ}$.

FIG. 2. - Fonctionnement du détecteur synchrone à la fréquence de $30 \mathrm{~Hz}$.

\section{Bibliographie}

[1] Schuster (N. A.), Rev. of Scient. Inst., 1951, 22, [4] Arecchi (F. T.), Gatti(E.) et Sona (A.), Rev. of Scient. 254.

[2] Moore (R. D.), Electronics, 1962, 35, 40.

[3] Faulkner (E. A.) et Stannet (R. H. O.), Electronic Ingng., 1964, 36, 159.

Inst., 1966, 37, 942.

[5] Dicke (R. H.), Rev. of Scient. Inst., 1946, 17, 268.

[6] Robinson (F. N. H.), ( Noise in Electrical Circuits ), Oxford University Press, 1962. 